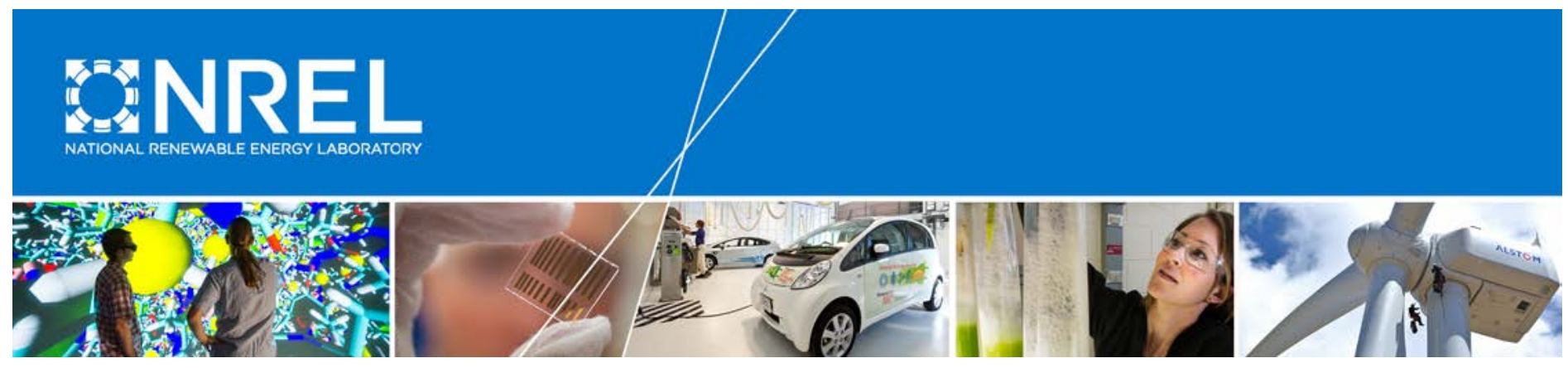

\title{
Comparison of Virtual Oscillator and Droop Control
}

\section{Preprint}

\author{
Brian Johnson and Miguel Rodriguez \\ National Renewable Energy Laboratory \\ Mohit Sinha and Sairaj Dhople \\ University of Minnesota
}

Presented at the IEEE COMPEL 2017

Stanford, California

June 9-12, 2017

(c) 2017 IEEE. Personal use of this material is permitted. Permission from IEEE must be obtained for all other uses, in any current or future media, including reprinting/republishing this material for advertising or promotional purposes, creating new collective works, for resale or redistribution to servers or lists, or reuse of any copyrighted component of this work in other works.

NREL is a national laboratory of the U.S. Department of Energy Office of Energy Efficiency \& Renewable Energy Operated by the Alliance for Sustainable Energy, LLC

This report is available at no cost from the National Renewable Energy Laboratory (NREL) at www.nrel.gov/publications.

\section{Conference Paper}

NREL/CP-5D00-68204

September 2017 


\section{NOTICE}

The submitted manuscript has been offered by an employee of the Alliance for Sustainable Energy, LLC (Alliance), a contractor of the US Government under Contract No. DE-AC36-08GO28308. Accordingly, the US Government and Alliance retain a nonexclusive royalty-free license to publish or reproduce the published form of this contribution, or allow others to do so, for US Government purposes.

This report was prepared as an account of work sponsored by an agency of the United States government. Neither the United States government nor any agency thereof, nor any of their employees, makes any warranty, express or implied, or assumes any legal liability or responsibility for the accuracy, completeness, or usefulness of any information, apparatus, product, or process disclosed, or represents that its use would not infringe privately owned rights. Reference herein to any specific commercial product, process, or service by trade name, trademark, manufacturer, or otherwise does not necessarily constitute or imply its endorsement, recommendation, or favoring by the United States government or any agency thereof. The views and opinions of authors expressed herein do not necessarily state or reflect those of the United States government or any agency thereof.

This report is available at no cost from the National Renewable Energy Laboratory (NREL) at www.nrel.gov/publications.

Available electronically at SciTech Connect http:/www.osti.gov/scitech

Available for a processing fee to U.S. Department of Energy and its contractors, in paper, from:

U.S. Department of Energy

Office of Scientific and Technical Information

P.O. Box 62

Oak Ridge, TN 37831-0062

OSTI http://www.osti.gov

Phone: 865.576.8401

Fax: 865.576.5728

Email: reports@osti.gov

Available for sale to the public, in paper, from:

U.S. Department of Commerce

National Technical Information Service

5301 Shawnee Road

Alexandria, VA 22312

NTIS http://www.ntis.gov

Phone: 800.553 .6847 or 703.605 .6000

Fax: 703.605.6900

Email: orders@ntis.gov 


\section{Comparison of Virtual Oscillator and Droop Control}

\author{
Brian Johnson, Miguel Rodriguez \\ Power Systems Engineering Center \\ National Renewable Energy Laboratory \\ Golden, CO 80401 \\ Email: brian.johnson@ nrel.gov, miguelrg@gmail.com
}

\author{
Mohit Sinha, Sairaj Dhople \\ Department of Electrical \& Computer Engineering \\ University of Minnesota \\ Minneapolis, MN 55455 \\ Email: $\{$ sinha052,sdhople $\} @$ umn.edu
}

\begin{abstract}
Virtual oscillator control (VOC) and droop control are distinct methods to ensure synchronization and power sharing of parallel inverters in islanded systems. VOC is a control strategy where the dynamics of a nonlinear oscillator are used to derive control states to modulate the switch terminals of an inverter. Since VOC is a time-domain controller that reacts to instantaneous measurements with no additional filters or computations, it provides a rapid response during transients and stabilizes volatile dynamics. In contrast, droop control regulates the inverter voltage in response to the measured average real and reactive power output. Given that real and reactive power are phasor quantities that are not well-defined in real time, droop controllers typically use multiplicative operations in conjunction with low-pass filters on the current and voltage measurements to calculate such quantities. Since these filters must suppress low frequency ac harmonics, they typically have low cutoff frequencies that ultimately impede droop controller bandwidth. Although VOC and droop control can be engineered to produce similar steady-state characteristics, their dynamic performance can differ markedly. This paper presents an analytical framework to characterize and compare the dynamic response of VOC and droop control. The analysis is experimentally validated with three $120 \mathrm{~V}$ inverters rated at $1 \mathrm{~kW}$, demonstrating that for the same design specifications VOC is roughly 8 times faster and presents almost no overshoot after a transient.
\end{abstract}

\section{INTRODUCTION}

Ac microgrids based on electronics generally contain multiple inverter interfaced energy sources connected across an ac bus where the system of inverters must collectively maintain system voltage and frequency. To enhance modularity and resilience to failures, decentralized controllers are generally preferred such that inverters can be added and removed as necessary and load sharing without communication is guaranteed. In this work, we examine the performance of two decentralized controllers for inverter-based microgrids: droop control and virtual oscillator control (VOC). Droop control is a classic method that draws inspiration from synchronous machine systems [1]-[4] and VOC is a recently developed approach that leverages contemporary advances in nonlinear control [5]. The main objective of this work is to compare the dynamic response of these two control strategies and uncover performance gains that one strategy may have over the other. Towards that end, we establish an analytical framework to assess the eigenvalues of both VOC and droop. After showing

Funding support from the the U.S. Department of Energy (DOE) Solar Energy Technologies Office under Contract No. DE-EE0000-1583, and the National Science Foundation under the CAREER award, ECCS-1453921, and grant ECCS-1509277 is gratefully acknowledged. that the linearized response of VOC indicates a more rapid and damped response in comparison to droop control, we further substantiate this result with an experimental setup consisting of three parallel inverters serving load. In particular, measurements show that VOC can synchronize roughly 8 times faster than droop for the particular hardware setup and design considered here.

At its core, the operation principle behind droop control is the enforcement of a linear relationship between measured real and reactive power with respect to frequency and voltage, respectively, at the inverter terminals. Since real and reactive power are not well-defined in real time and are generally assumed to be averaged quantities, we refer to droop control as being a phasor-domain controller to emphasize the fact that the control variables are essentially averaged phasors. From an implementation standpoint, this implies that computations from sinusoidal measurements must undergo processing and filtering to obtain the phasor quantities that form the basis of the droop control law. For instance, algebraic relationships are typically used to first obtain a preliminary set of real and reactive power values from the raw voltage and current measurements. However, because these algebraically computed values are susceptible to line frequency harmonics and distortion, they must be filtered before obtaining frequency and voltage commands via the linear droop laws. It should be noted that if the aforementioned filters were bypassed, any harmonics from the sinusoidal measurements would propagate to the controlled inverter voltage. So although the utilization of filters blocks undesirable harmonics from reaching the voltage and frequency commands, it should be clear that these filters form a key component of the control feedback path and may act as a performance bottleneck and lead to a sluggish response. To provide some context, it is not uncommon to see droop control filter cutoff frequencies below $10 \mathrm{~Hz}$. Taken together, this indicates that droop controllers exhibit a fundamental tradeoff between immunity to harmonics and control bandwidth.

VOC is a method that is based on digitally emulating a nonlinear oscillator circuit such that measurements act as an inputs to the oscillator and its states are used to modulate the inverter terminals directly. In contrast to droop control, VOC is a time-domain controller that acts on the real-time sinusoidal current delivered by the inverter such that it reacts nearly instantaneously to system disturbances without the need for additional filters or averaging. While VOC may appear to be a highly unconventional approach, recent work has shown that its steady-state behavior exhibit a droop-like 
characteristic between frequency and voltage with respect to real and reactive power. [6] In essence, VOC subsumes the functionality of droop in steady-state while providing superior speed during transients. Furthermore, fast synchronization for parallel-connected inverters by using oscillator based nonlinear control was also reported in [7] where the authors also pointed out the limitations of the phasor-based methods for control during transients.

While the enhanced speed of VOC over droop has been previously reported via simulation [8], here we take the first step at analytically characterizing such differences and providing an experimental validation. In Section II we outline foundations of the inverter model. Section III outlines the dynamical models of droop control and VOC as well as their corresponding linearized representations. Simulation and experimental results are given in Section IV and concluding statements are in Section V.

\section{PRELIMINARIES}

We base our analysis on the inverter models in Fig. 1. In each case, an inverter is connected to bus voltage, $v_{\mathrm{b}}$, with RMS amplitude $V_{\mathrm{b}}$, instantaneous angle $\theta_{\mathrm{b}}$, and frequency $\omega_{\mathrm{b}}=\dot{\theta}_{\mathrm{b}}$ (i.e., $\left.v_{\mathrm{b}}=\sqrt{2} V_{\mathrm{b}} \cos \theta_{\mathrm{b}}\right)$. Ultimately, each controller produces a sinusoidal voltage command of the form, $v=\sqrt{2} V \cos \theta_{\mathrm{i}}$, where $V$ and $\theta_{\mathrm{i}}$ are the terminal RMS voltage amplitude and angle, respectively. The modulation signal is derived from the voltage command via $m=v / v_{\mathrm{dc}}$ and pulse width modulation is applied to obtain the switch states where $\widetilde{v}$ denotes the voltage across the switch terminals. From here forward, we utilize an averaged value model where it is assumed that the switch terminal voltage perfectly tracks the commanded value such that $\widetilde{v}=v$. This can be justified under the setting where $m$ varies on a much slower timescale in comparison to the switch period, $T_{\mathrm{sw}}$, [9] and the following expression holds

$$
\frac{1}{T_{\mathrm{sw}}} \int_{s=t-T_{\mathrm{sw}}}^{t} \widetilde{v}(s) d s=m(t) v_{\mathrm{dc}}=v(t) .
$$

Subsequent analysis is carried out in the inverter reference $d q$ frame which rotates with angle $\theta_{\mathrm{i}}$. To facilitate analysis, we will use the inverter-bus angle difference, denoted as $\delta=\theta_{\mathrm{i}}-\theta_{\mathrm{b}}$, rather than the inverter and bus angles themselves. This choice is motivated by the fact that while the instantaneous angles grow continuously, their difference reaches an equilibrium if the system reaches steady-state. Since the model is in the inverter reference frame, we note that $\left[v_{d}, v_{q}\right]^{\top}=[V, 0]^{\top}$, where $v_{d}$ and $v_{q}$ are the $d q$ components of the inverter terminal voltage. The $R L$ output filter has an inductance and resistance of $L_{\mathrm{f}}$ and $R_{\mathrm{f}}$ and the $d q$ components of the current flowing through this branch are denoted as $i_{d}$ and $i_{q}$. The state equations for the current and inverter-bus angle difference are [10]

$$
\begin{aligned}
\frac{d}{d t}\left[\begin{array}{l}
i_{d} \\
i_{q}
\end{array}\right] & =\left[\begin{array}{cc}
-\frac{R_{\mathrm{f}}}{L_{\mathrm{f}}} & \omega_{\mathrm{i}} \\
-\omega_{\mathrm{i}} & -\frac{R_{\mathrm{f}}}{L_{\mathrm{f}}}
\end{array}\right]\left[\begin{array}{l}
i_{d} \\
i_{q}
\end{array}\right]+\left[\begin{array}{c}
V-v_{\mathrm{b} d} \\
-v_{\mathrm{b} q}
\end{array}\right], \\
\frac{d \delta}{d t} & =\omega_{i}-\omega_{b} .
\end{aligned}
$$

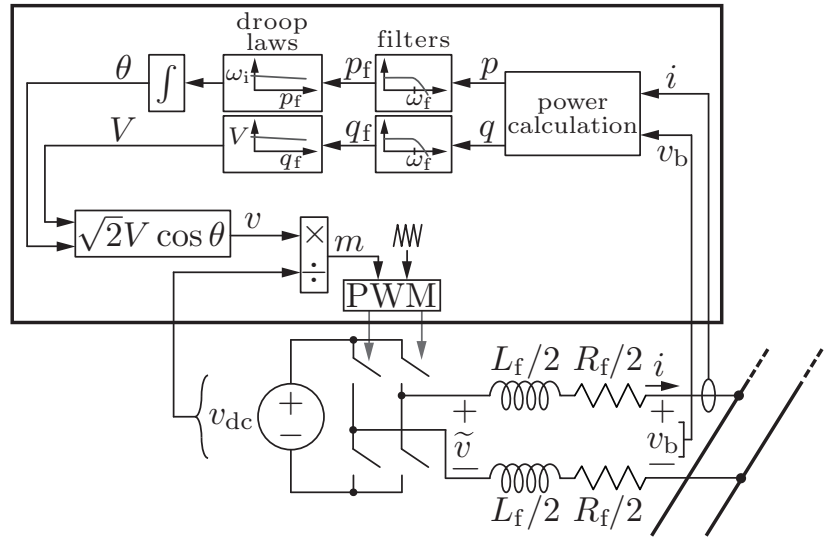

(a)

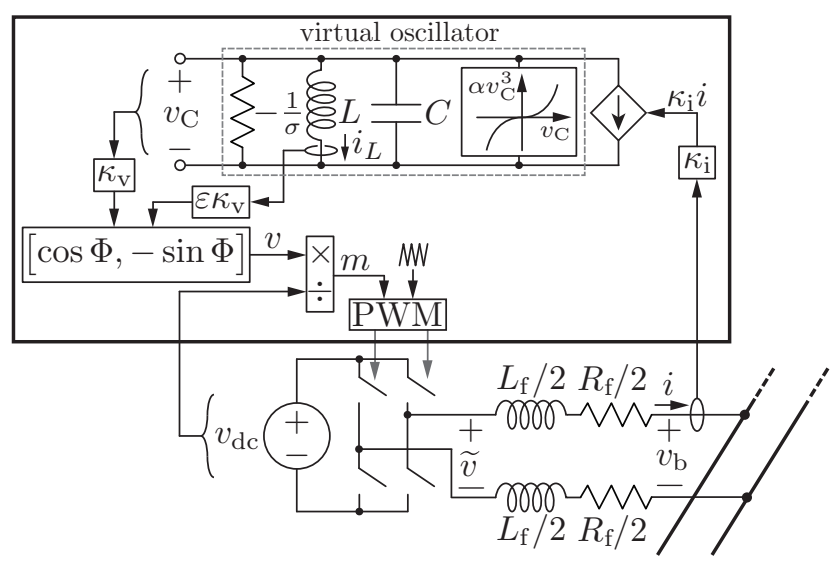

(b)

Fig. 1: Typical implementations of droop controlled and VO-controlled inverters are given in (a) and (b), respectively. In the subsequent analysis, we adopt an averaged value model where we assume that the voltage across the switch terminals, $\widetilde{v}$, follows the control voltage $v(t)$.

The state equations in (2)-(3) are of particular importance since they will be used for both models in Fig. 1. Note that although the analysis here can be easily extended to more complex filter structures, we restrict our focus to the $R L$ filter. The bus voltages, $v_{\mathrm{b} d}$ and $v_{\mathrm{b} q}$ in (2), which are specified in the inverter reference frame, can be obtained via the following transformation

$$
\left[\begin{array}{c}
v_{\mathrm{b} d} \\
v_{\mathrm{b} q}
\end{array}\right]=\left[\begin{array}{cc}
\cos \left(\theta_{\mathrm{i}}-\theta_{\mathrm{b}}\right) & \sin \left(\theta_{\mathrm{i}}-\theta_{\mathrm{b}}\right) \\
-\sin \left(\theta_{\mathrm{i}}-\theta_{\mathrm{b}}\right) & \cos \left(\theta_{\mathrm{i}}-\theta_{\mathrm{b}}\right)
\end{array}\right]\left[\begin{array}{c}
v_{\mathrm{b} D} \\
v_{\mathrm{b} Q}
\end{array}\right],
$$

where $v_{\mathrm{b} D}$ and $v_{\mathrm{b} D}$ are the $\mathrm{D}$ and $\mathrm{Q}$ components of the bus voltage in its own frame rotating at angle $\theta_{\mathrm{b}}$. Then, without loss of generality, we can specify $\left[v_{\mathrm{b} D}, v_{\mathrm{b} Q}\right]^{\top}=\left[V_{\mathrm{b}}, 0\right]^{\top}$.

\section{Modeling OF CONTROLled InVERTERS}

\section{A. Droop control}

A single inverter with droop control is illustrated in Fig. 1(a). The first stage of the controller is a power computation which uses the filter current and bus voltage, $i$ and $v_{\mathrm{b}}$, to compute the instantaneous real and reactive power, $p$ and $q$, respectively. To remove ac harmonics, these signals are processed by a first-order low-pass filter to obtain the filtered signals $p_{\mathrm{f}}$ and $q_{\mathrm{f}}$. Lastly, the droop laws yield the voltage amplitude, $V$, and frequency, $\omega_{i}$, that are used to construct the switch terminal voltage waveform, $v=\sqrt{2} V \cos \theta_{\mathrm{i}}$, where 
$\theta_{\mathrm{i}}$ is obtained by integrating $\omega_{\mathrm{i}}$. The instantaneous real and reactive power computations and droop laws can be specified in the inverter frame $d q$ coordinates as

$$
\begin{aligned}
{\left[\begin{array}{c}
p \\
q
\end{array}\right] } & =\left[\begin{array}{cc}
v_{\mathrm{b} d} & v_{\mathrm{b} q} \\
v_{\mathrm{b} q} & -v_{\mathrm{b} d}
\end{array}\right]\left[\begin{array}{c}
i_{d} \\
i_{q}
\end{array}\right], \\
V & =V_{\mathrm{nom}}-m_{\mathrm{q}}\left(q_{\mathrm{f}}-q^{*}\right), \\
\omega_{\mathrm{i}} & =\omega_{\mathrm{nom}}-m_{\mathrm{p}}\left(p_{\mathrm{f}}-p^{*}\right),
\end{aligned}
$$

where $V_{\text {nom }}$ and $\omega_{\text {nom }}$ are the nominal voltage and frequency while $p^{*}$ and $q^{*}$ are the associated setpoints. The voltage and frequency droop slopes are $m_{\mathrm{q}}$ and $m_{\mathrm{p}}$. The state equations that are unique to the droop controlled inverter are

$$
\frac{d}{d t}\left[\begin{array}{c}
p_{\mathrm{f}} \\
q_{\mathrm{f}}
\end{array}\right]=-\omega_{\mathrm{f}}\left[\begin{array}{c}
p_{\mathrm{f}} \\
q_{\mathrm{f}}
\end{array}\right]+\left[\begin{array}{c}
p \\
q
\end{array}\right],
$$

where $\omega_{\mathrm{f}}$ is the cutoff frequency for the active and reactive power filter. Considering the inverter states in Section II, the overall droop system in Fig. 1(a) has the following states: $p_{\mathrm{f}}, q_{\mathrm{f}}, i_{d}, i_{q}$, and $\delta$.

The expressions in (2), (3), and (8) specify the non-linear dynamics of a single droop-controlled inverter connected to a voltage bus. To gain insights on the dynamic response of the system, we linearize these expressions to obtain the following small-signal model:

$$
\begin{aligned}
\frac{d}{d t}\left[\begin{array}{c}
\Delta p_{\mathrm{f}} \\
\Delta q_{\mathrm{f}} \\
\Delta \delta \\
\Delta i_{d} \\
\Delta i_{q}
\end{array}\right]= & {\left[\begin{array}{ccccc}
-\omega_{\mathrm{f}} & 0 & 0 & V_{\mathrm{b}} \omega_{\mathrm{f}} & 0 \\
0 & -\omega_{\mathrm{f}} & 0 & 0 & -V_{\mathrm{b}} \omega_{\mathrm{f}} \\
-m_{\mathrm{p}} & 0 & 0 & 0 & 0 \\
0 & -\frac{m_{\mathrm{q}}}{L_{\mathrm{f}}} & 0 & -\frac{R_{\mathrm{f}}}{L_{\mathrm{f}}} & \bar{\omega}_{\mathrm{i}} \\
0 & 0 & \frac{V_{\mathrm{b}}}{L_{\mathrm{f}}} & -\bar{\omega}_{\mathrm{i}} & -\frac{R_{\mathrm{f}}}{L_{\mathrm{f}}}
\end{array}\right]\left[\begin{array}{c}
\Delta p_{\mathrm{f}} \\
\Delta q_{\mathrm{f}} \\
\Delta \delta \\
\Delta i_{d} \\
\Delta i_{q}
\end{array}\right] } \\
& +\left[\begin{array}{lllll}
0 & 0 & -1 & 0 & 0
\end{array}\right]^{\top} \Delta \omega_{\mathrm{b}},
\end{aligned}
$$

where $\bar{\omega}_{\mathrm{i}}$ is the inverter frequency at equilibrium, the equilibrium currents $\bar{i}_{d}$ and $\bar{i}_{q}$ are set to zero, the equilibrium angle difference is assumed to be negligible, and the bus frequency deviation, $\Delta \omega_{\mathrm{b}}$, is interpreted as a model input. The negligible angle difference approximation is reasonable to study the response to a system transient since steady-state angle differences just before a transient are typically small. ${ }^{1}$ In the context of this model, system disturbances, such as the addition and removal of inverters, will be interpreted as a change in bus frequency. This framework can be extended to accommodate bus voltage disturbances. Finally, note that the setpoints $p^{*}$ and $q^{*}$ have been set to 0 . From (9), we can derive the characteristic equation, as given below, whose roots give us the system eigenvalues:

$$
\begin{aligned}
D(s) & =L_{\mathrm{f}}^{2} s^{5}+\left(2 \omega_{\mathrm{f}} L_{\mathrm{f}}^{2}+2 R_{\mathrm{f}}\right) s^{4} \\
& +\left(L_{\mathrm{f}}^{2} \omega_{\mathrm{f}}^{2}+L_{\mathrm{f}}^{2} \bar{\omega}_{\mathrm{i}}^{2}+4 L_{\mathrm{f}} \omega_{\mathrm{f}} R_{\mathrm{f}}+R_{\mathrm{f}}^{2}\right) s^{3} \\
& +\left(2 L_{\mathrm{f}}^{2} \omega_{\mathrm{f}} \bar{\omega}_{\mathrm{i}}^{2}+2 L_{\mathrm{f}} \omega_{\mathrm{f}}^{2} R_{\mathrm{f}}+V_{\mathrm{b}} m_{\mathrm{q}} L_{\mathrm{f}} \omega_{\mathrm{f}} \bar{\omega}_{\mathrm{i}}+2 \omega_{\mathrm{f}} R_{\mathrm{f}}^{2}\right) s^{2} \\
& +\left(L_{\mathrm{f}}^{2} \omega_{\mathrm{f}}^{2} \bar{\omega}_{\mathrm{i}}^{2}+m_{\mathrm{p}} L_{\mathrm{f}} V_{\mathrm{b}}^{2} \omega_{\mathrm{f}} \bar{\omega}_{\mathrm{i}}+m_{\mathrm{q}} L_{\mathrm{f}} V_{\mathrm{b}} \omega_{\mathrm{f}}^{2} \bar{\omega}_{\mathrm{i}}+\omega_{\mathrm{f}}^{2} R_{\mathrm{f}}^{2}\right) s \\
& +m_{\mathrm{q}} m_{\mathrm{p}} V_{\mathrm{b}}^{3} \omega_{\mathrm{f}}^{2}+L_{\mathrm{f}} m_{\mathrm{p}} \bar{\omega}_{\mathrm{i}} V_{\mathrm{b}}^{2} \omega_{\mathrm{f}}^{2} .
\end{aligned}
$$

Here we note that the linearized formulation in (9) is similar to that used in [11]-[14], but with a reduced number of states

\footnotetext{
${ }^{1}$ Note that the linearized model can be straightforwardly linearized around non-zero currents. Here we set them to zero as it was observed that their value made a trivial impact on the numerical results.
}

to facilitate the calculation of (10). Furthermore, our result in (10) is obtained from first principles as opposed to the more complex methods used in [15].

\section{B. Dynamic Model of Virtual Oscillator Control}

The dynamics of the states within the virtual oscillator are given by

$$
\begin{aligned}
L \frac{d i_{L}}{d t} & =v_{C} \\
C \frac{d v_{C}}{d t} & =-\alpha v_{C}^{3}+\sigma v_{C}-i_{L}-\kappa_{\mathrm{i}} i
\end{aligned}
$$

where $v_{C}$ and $i_{L}$ are the virtual capacitor voltage and inductor current, respectively. The virtual capacitance, inductance, and conductance are given by $C, L$, and $\sigma$, and the nonlinear voltage dependent current source is parameterized by $\alpha$. The controller is interfaced via voltage and current scaling values, $\kappa_{\mathrm{V}}$ and $\kappa_{\mathrm{i}}$. As illustrated in Fig. 1(b), the output voltage command is given by

$$
v=\kappa_{\mathrm{v}}\left(v_{C} \cos \Phi-\varepsilon i_{L} \sin \Phi\right),
$$

where $\varepsilon=\sqrt{L / C}$ and $\Phi$ is a fixed user-defined angle, typically chosen as either 0 or $\pi / 2$, that specifies the type of droop-like characteristic the VO-controlled inverter exhibits in steady-state.

Since VOC is a time-domain controller and its implementation in Fig. 1 offers no clear notion of phasor quantities such as amplitude, angle, frequency, and power, we must utilize an averaged model that is written in these terms in order to enable a comparison with the droop control. As delineated in [16], such a model can be obtained by averaging the trajectory of the oscillator in Fig. 1(b) over one ac cycle such that we obtain the following amplitude and frequency dynamics

$$
\begin{aligned}
& \frac{d V}{d t}=\frac{\sigma}{2 C}\left(V-\frac{\beta}{2} V^{3}\right)-\frac{\kappa_{\mathrm{i}} \kappa_{\mathrm{v}}}{2 C V} q, \\
& \frac{d \theta_{\mathrm{i}}}{d t}=\omega_{\mathrm{i}}=\omega_{\text {nom }}-\frac{\kappa_{\mathrm{i}} \kappa_{\mathrm{v}}}{2 C V^{2}} p,
\end{aligned}
$$

where $\beta=3 \alpha\left(\kappa_{\mathrm{v}}^{2} \sigma\right)^{-1}$ and the following model is obtained with $\Phi=\pi / 2$ such that the VOC system exhibits $V$-versus- $q$ and $\omega_{\mathrm{i}}$-versus- $p$ droop-like relationships [8] in alignment with the form of the conventional droop controller considered in Section III-A. Although we use $\Phi=\pi / 2$ to ensure a similar steady-state relationship between the two controllers under consideration, it should be pointed out that $p$ and $q$ in (13)-(14) are computed using unfiltered signals and are given by

$$
\left[\begin{array}{l}
p \\
q
\end{array}\right]=\left[\begin{array}{cc}
V & 0 \\
0 & -V
\end{array}\right]\left[\begin{array}{l}
i_{d} \\
i_{q}
\end{array}\right] .
$$

This follows from the fact that VOC is a time-domain controller and its averaged formulation, as we consider here, naturally incorporates a one-cycle moving average without any additional filters or computations. Note that (5) and (15) take on slightly different form. This discrepancy is due to the fact that whereas droop control typically uses the measured real and reactive power at the bus terminals, the physical structure of the VOC feedback loop in Fig. 1(b) naturally implies a power computation at the switch terminals.

After substituting (14) into (3), the expressions in (2), (13) and the inverter-bus angle difference can be linearized around 
Table I: Parameters of the inverter hardware, droop controller in Section III-A, and oscillator-based controller in Section III-B.

\begin{tabular}{c|c|c}
\hline$L_{\mathrm{f}}=1 \mathrm{mH}$ & $R_{\mathrm{f}}=0.7 \Omega$ & $L_{\mathrm{o}}=0.2 \mathrm{mH}$ \\
\hline$R_{\mathrm{o}}=0.12 \Omega$ & $C_{\mathrm{f}}=24 \mu \mathrm{F}$ & $V_{\mathrm{dc}}=220 \mathrm{~V}$ \\
\hline$S_{\text {nom }}=750 \mathrm{VA}$ & $V_{\text {nom }}=120 \mathrm{~V}$ & $\omega_{\text {nom }}=2 \pi 60 \mathrm{rad} / \mathrm{s}$ \\
\hline \hline$m_{\mathrm{p}}=\frac{2 \pi 0.5 \mathrm{rad} / \mathrm{s}}{S_{\text {nom }}}$ & $m_{\mathrm{q}}=0.05 \frac{V_{\text {nom }}}{S_{\text {nom }}}$ & $\omega_{\mathrm{f}}=2 \pi 5 \mathrm{rad} / \mathrm{s}$ \\
\hline \hline$L=39.9 \mu \mathrm{H}$ & $C=176.3 \mathrm{mF}$ & $\sigma=11.4 \Omega^{-1}$ \\
\hline$\alpha=7.58 \mathrm{~A} / \mathrm{V}^{3}$ & $\kappa_{\mathrm{V}}=120 \mathrm{~V} / \mathrm{V}$ & $\kappa_{\mathrm{i}}=0.16 \mathrm{~A} / \mathrm{A}$ \\
\hline
\end{tabular}

the steady-state values, $\bar{V}, \bar{p}$, and $\bar{q}$. This yields the following representation

$$
\begin{aligned}
\frac{d}{d t}\left[\begin{array}{c}
\Delta \delta \\
\Delta i_{d} \\
\Delta i_{q} \\
\Delta V
\end{array}\right]= & {\left[\begin{array}{cccc}
0 & -\frac{\kappa_{\mathrm{i}} \kappa_{\mathrm{v}}}{2 C \bar{V}} & 0 & 0 \\
0 & -\frac{R_{\mathrm{f}}}{L_{\mathrm{f}}} & \omega_{\mathrm{nom}} & \frac{1}{L_{\mathrm{f}}} \\
\frac{V_{\mathrm{b}}}{L_{\mathrm{f}}} & -\omega_{\text {nom }} & -\frac{R_{\mathrm{f}}}{L_{\mathrm{f}}} & 0 \\
0 & 0 & \frac{\kappa_{\mathrm{i}} \kappa_{\mathrm{v}}}{2 C} & \chi
\end{array}\right]\left[\begin{array}{c}
\Delta \delta \\
\Delta i_{d} \\
\Delta i_{q} \\
\Delta V
\end{array}\right] } \\
& +\left[\begin{array}{llll}
-1 & 0 & 0 & 0
\end{array}\right]^{\top} \Delta \omega_{\mathrm{b}}
\end{aligned}
$$

where $\chi=\sigma\left(1-3 \beta \bar{V}^{2} / 2\right) /(2 C)$ and again we assume the current and angle differences at equilibrium are negligible. Note that the VOC system has one less state in comparison to droop since it does not contain low-pass filters and those associated states but has one additional voltage dynamic state given by (13). The characteristic equation for the VOC system takes the following form:

$$
\begin{aligned}
D(s)= & \left(8 C^{2} L_{\mathrm{f}}^{2} \bar{V}\right) s^{4} \\
+ & \left(16 R_{\mathrm{f}} C^{2} L_{\mathrm{f}} \bar{V}+6 \beta \sigma C L_{\mathrm{f}}^{2} \bar{V}^{3}-4 \sigma C L_{\mathrm{f}}^{2} \bar{V}\right) s^{3} \\
+ & \left(8 C^{2} L_{\mathrm{f}}^{2} \bar{V} \omega_{\text {nom }}+8 C^{2} \bar{V} R_{\mathrm{f}}^{2}\right. \\
& \left.+12 \beta \sigma C L_{\mathrm{f}} \bar{V}^{3} R_{\mathrm{f}}-8 \sigma C L_{\mathrm{f}} \bar{V} R_{\mathrm{f}}\right) s^{2} \\
+ & \left(6 C \beta \sigma V^{3} L_{\mathrm{f}}^{2} \omega_{\text {nom }}^{2}-4 C \sigma \bar{V} L_{\mathrm{f}}^{2} \omega_{\text {nom }}^{2}\right. \\
& +4 C \kappa_{i} \kappa_{\mathrm{v}} L_{\mathrm{f}} \bar{V} \omega_{\text {nom }}+4 C V_{\mathrm{b}} \kappa_{\mathrm{i}} \kappa_{\mathrm{v}} L_{\mathrm{f}} \omega_{\text {nom }} \\
& \left.+6 C \beta \sigma \bar{V}^{3} R_{\mathrm{f}}^{2}-4 C \sigma \bar{V} R_{\mathrm{f}}^{2}\right) s \\
+ & 3 L_{\mathrm{f}} V_{\mathrm{b}} \beta \sigma \omega_{\text {nom }} \bar{V}^{2} \kappa_{\mathrm{i}} \kappa_{\mathrm{v}}+2 V_{\mathrm{b}} \kappa_{\mathrm{i}}^{2} \kappa_{\mathrm{v}}^{2} \\
- & 2 L_{\mathrm{f}} V_{\mathrm{b}} \sigma \omega_{\text {nom }} \kappa_{\mathrm{i}} \kappa_{\mathrm{v}} .
\end{aligned}
$$

\section{Simulation AND Experimental Results}

In this section, we first present numerical results based on the analysis in Sections III-A and III-B. Thereafter, we compare the measured response of a hardware system under a transient for both droop control and VOC. In all the ensuing analysis, the numerical paramaters are based on the experimental hardware for a hardware prototype rated at $1 \mathrm{kVA}$ and 120 V RMS.

\section{A. Eigenvalue analysis}

Here we assess the roots of (10) and (17) as we sweep certain parameters from the nominal values specified in Table I. Figures 2(a) and 2(b) show the roots of (10) and (17) as a function of the inverter output inductance and resistance, $L_{\mathrm{f}}$ and $R_{\mathrm{f}}$. For the linearized operating conditions, we assume

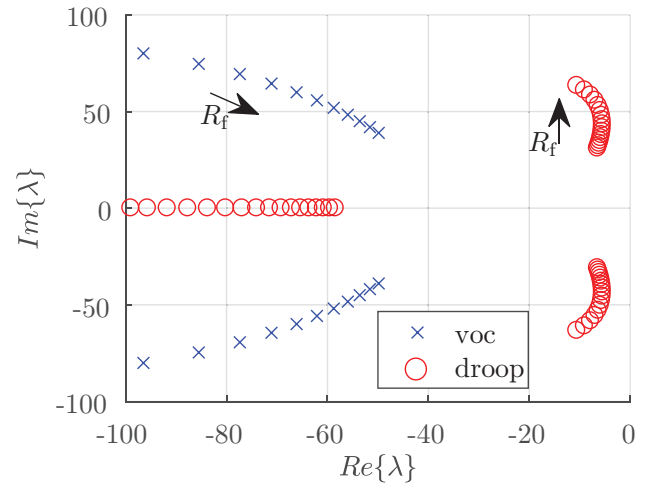

(a)

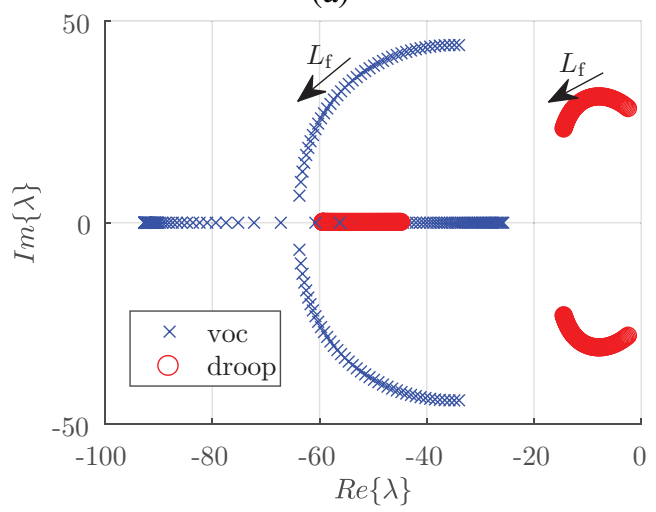

(b)

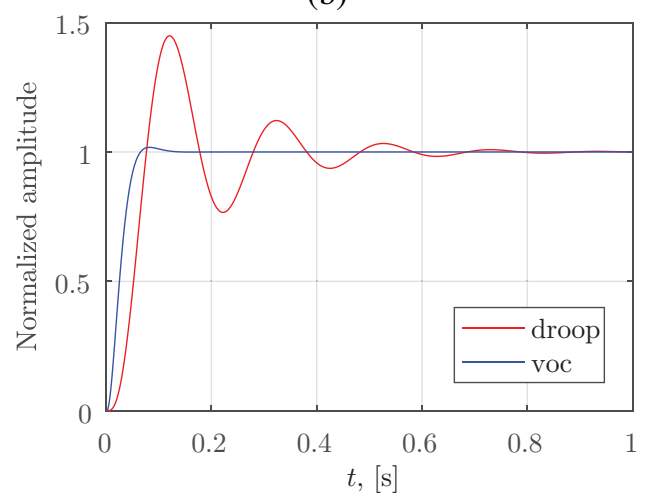

(c)

Fig. 2: (a) Root locus of (10) and (17) for $R_{\mathrm{f}}=0.1 \Omega \rightarrow 1 \Omega$. (b) Root locus of (10) and (17) for $L_{\mathrm{f}}=0.1 \mathrm{mH} \rightarrow 1 \mathrm{mH}$. (c) Unit step response of the transfer function $1 / D(s)$.

$V_{\mathrm{b}}=120 \mathrm{~V}$ and $\bar{\omega}_{i}=2 \pi 60 \mathrm{rad} / \mathrm{s}$. Furthermore, $m_{\mathrm{q}}$ and $m_{\mathrm{p}}$ are chosen to achieve a maximum $5 \%$ frequency and voltage variation over the full-rated operating regime for both for droop and VOC (see [8], [16] for how $m_{\mathrm{p}}$ and $m_{\mathrm{q}}$ can be translated to VOC parameters for equivalent steady-state performance). In Figs. 2(a)-(b), it is clear that the smallest negative real parts of the VOC eigenvalues are consistently farther away from the imaginary axis in comparison to those of the linearized droop system. This implies a faster and more damped response for VOC. The normalized step response of $1 / D(s)$ response in Fig. 2(c) corroborates this intuition.

\section{B. Hardware validation}

A setup consisting of three $1 \mathrm{kVA}$ parallel inverters operated at $120 V_{\text {rms }}$ in an islanded configuration was used to validate 


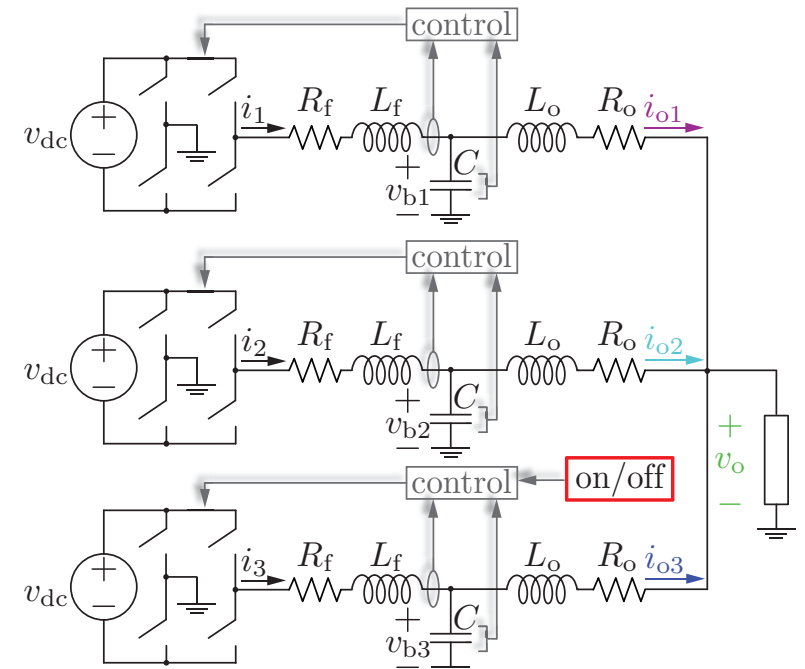

Fig. 3: Diagram of experimental setup consisting of three parallel connected inverters serving a load. To compare the performance of the oscillator- and droop-based controllers, we subject the system to identical transient conditions under each control strategy and assess their dynamic performance. For both tests, the system is first energized with two inverters and once they reach steady state the third inverter is turned on abruptly.

the analytical findings. Each unit exhibited the physical characteristics in Table I, was controlled with a TI F28335 DSP, and utilized an $L C L$ output filter where $C_{\mathrm{f}}$ denotes the filter capacitance, and the grid-side branch inductance and resistance are denoted as $L_{\mathrm{o}}$ and $r_{\mathrm{o}}$, respectively (see Fig. 3 to clarify the notation of the filter parameters). Note that it can be shown via frequency-domain analysis that the component values under consideration allow us to approximate the $L C L$ filter by a simple $L R$ filter with relatively high accuracy up to $500 \mathrm{~Hz}$. Accordingly, the analysis in prior sections retains sufficient accuracy for the actual experimental setup and for the timescales of interest. To reiterate, the controllers were both designed for equivalent performance such that $\omega_{\text {nom }}=2 \pi 60 \mathrm{rad} / \mathrm{s}, m_{\mathrm{p}}$ and $m_{\mathrm{q}}$ were identical for both droop control and VOC. Figures 4(a)-(b) show the oscilloscope captures of the transient after energizing the power semiconductors of the third unit in the system for $\mathrm{VO}$ and droop controlled inverters, respectively. To elaborate further, the controller for inverter \#3 was configured to synchronize its internal states to the measured terminal voltage and wait for a zero crossing before engaging the gate signals. This procedure was utilized for both controllers and was motivated by the need to avoid destructive transient currents during turn-on.

Visually inspecting the results in Fig. 4, it is readily apparent that VOC is substantially faster than VOC. To further elucidate the performance gains of VOC and obtain a concrete metric between both systems, we employ the projector matrix [5] to quantify the synchronization error, which is denoted and defined as $\Pi:=I_{N}-\mathbf{1}_{N} \mathbf{1}_{N}^{\top} / N$, where $N$ is the number of operational inverters, $I_{N}$ is an $N \times N$ identity matrix and $\mathbf{1}_{N}$ is a $N \times 1$ column-vector of ones. As shown in [17], the Euclidean norm $\|\Pi x\|_{2}$ is non-negative and is proportional to the proportional differences between each and every element in the $N \times 1$ vector $x$. Hence, because $\|\Pi x\|_{2}$ approaches zero only in the case when each element is synchronized,

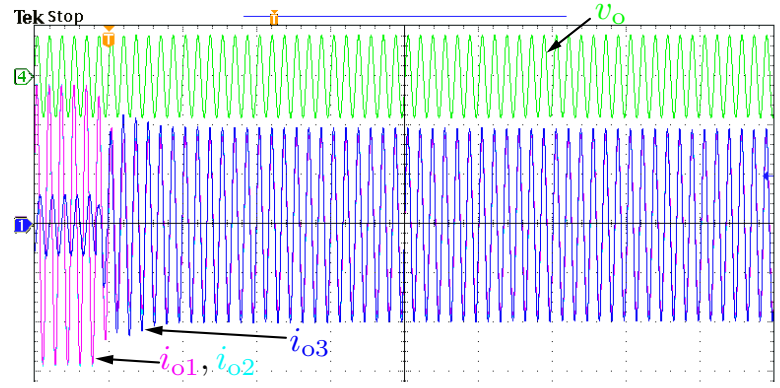

(a)

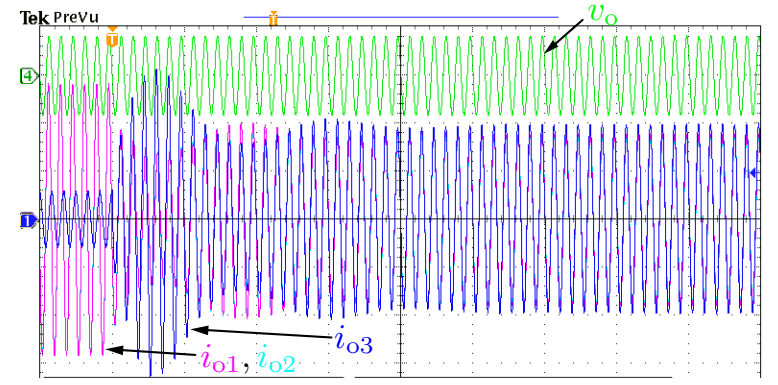

(b)

Fig. 4: Addition of inverter 3 when inverters 1 and 2 are supplying a $1 \mathrm{~kW}$ load. In each plot, the timescale is $100 \mathrm{~ms} / \mathrm{div}, 2 \mathrm{~A} / \mathrm{div}$ (for $i_{\mathrm{o} 1}, i_{\mathrm{o} 2}, i_{\mathrm{o} 3}$ ), and $100 \mathrm{~V} / \mathrm{div}$ for $v_{g}$. (a) and (b) show the response of the inverter system with VOC and droop control, respectively. Here, the superior speed and transient response of VOC is evident.

we will use it to quantify the synchronization error between multiple inverters on a system. Referring to Fig. 5, we plot the synchronization error in the vector of measured output currents $i_{\mathrm{o}}=\left[i_{\mathrm{o} 1}, i_{\mathrm{o} 2}, i_{\mathrm{o} 3}\right]^{\top}$. As illustrated, the VOC and droop-controlled systems fall below and remain under the error threshold in $45 \mathrm{~ms}$ and $346 \mathrm{~ms}$, respectively, where the threshold is defined as the measured maximum steady-state error among both controllers and is equal to approximately $1.45 \mathrm{~A}$ for our system. Hence, VOC synchronizes approximately 7.7 (or roughly 8) times faster than droop for this particular design.

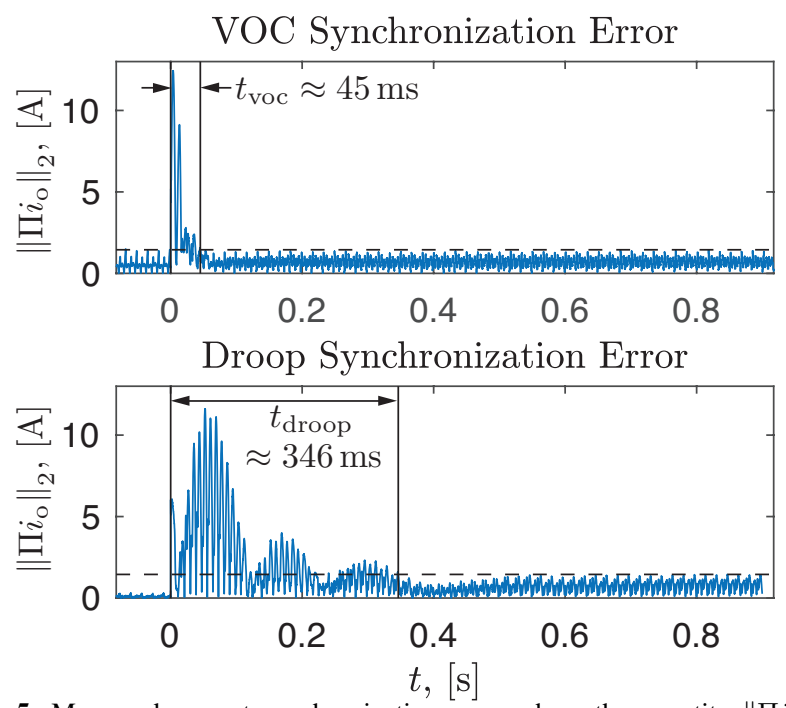

Fig. 5: Measured current synchronization error where the quantity $\left\|\Pi i_{\mathrm{o}}\right\|_{2}$ is proportional to the differences between the currents in the vector $i_{\mathrm{O}}=$ $\left[i_{\mathrm{o} 1}, i_{\mathrm{o} 2}, i_{\mathrm{o} 3}\right]^{\top}$. 


\section{Conclusions}

Droop and VOC are two methods to ensure power sharing and synchronization of parallel inverters in islanded microgrids. In this work, their dynamic performance was studied using a unified analytical framework. A dynamic model of each inverter system was developed and linearized to obtain their eigenvalues. Analytical and experimental results show that the droop method suffers from inherent limitations in its dynamic performance due to the need to measure and filter real and reactive power. In contrast, VOC acts on instantaneous measurements and inherently provides a faster and betterdamped response. Simulation and experimental results corroborate these findings and hardware measurements indicate a speed enhancement factor of approximately 7.7 with VOC for the particular design considered here.

\section{REFERENCES}

[1] M. C. Chandorkar, D. M. Divan, and R. Adapa, "Control of parallel connected inverters in stand-alone ac supply systems," in Conference Record of the 1991 IEEE Industry Applications Society Annual Meeting, pp. 1003-1009 vol.1, Sept 1991.

[2] Q. C. Zhong, "Robust droop controller for accurate proportional load sharing among inverters operated in parallel," IEEE Transactions on Industrial Electronics, vol. 60, pp. 1281-1290, April 2013.

[3] Q. C. Zhong and Y. Zeng, "Universal droop control of inverters with different types of output impedance," IEEE Access, vol. 4, pp. 702-712, 2016.

[4] Y. Sun, X. Hou, J. Yang, H. Han, M. Su, and J. M. Guerrero, "New perspectives on droop control in ac microgrid," IEEE Transactions on Industrial Electronics, vol. 64, pp. 5741-5745, July 2017.

[5] B. B. Johnson, S. V. Dhople, A. O. Hamadeh, and P. T. Krein, "Synchronization of parallel single-phase inverters with virtual oscillator control," IEEE Transactions on Power Electronics, vol. 29, pp. 61246138, Nov 2014.

[6] M. Sinha, F. Dörfler, B. B. Johnson, and S. V. Dhople, "Uncovering droop control laws embedded within the nonlinear dynamics of van der pol oscillators," IEEE Transactions on Control of Network Systems, vol. 4, pp. 347-358, June 2017.

[7] L. A. B. Tôrres, J. P. Hespanha, and J. Moehlis, "Synchronization of identical oscillators coupled through a symmetric network with dynamics: A constructive approach with applications to parallel operation of inverters," IEEE Transactions on Automatic Control, vol. 60, pp. 32263241, Dec 2015.

[8] M. Sinha, S. Dhople, B. Johnson, N. Ainsworth, and F. Dörfler, "Nonlinear supersets to droop control," in 2015 IEEE 16th Workshop on Control and Modeling for Power Electronics (COMPEL), pp. 1-6, July 2015.

[9] P. T. Krein, Elements of power electronics, vol. 101. Oxford University Press New York, 1998.

[10] A. Yazdani and R. Iravani, Voltage-sourced converters in power systems: modeling, control, and applications. John Wiley \& Sons, 2010.

[11] N. Pogaku, M. Prodanovic, and T. C. Green, "Modeling, analysis and testing of autonomous operation of an inverter-based microgrid," IEEE Transactions on Power Electronics, vol. 22, pp. 613-625, March 2007.

[12] N. Bottrell, M. Prodanovic, and T. C. Green, "Dynamic stability of a microgrid with an active load," IEEE Transactions on Power Electronics, vol. 28, pp. 5107-5119, Nov 2013.

[13] M. F. M. Arani and Y. A. R. I. Mohamed, "Analysis and impacts of implementing droop control in dfig-based wind turbines on microgrid/weak-grid stability," IEEE Transactions on Power Systems, vol. 30, pp. 385-396, Jan 2015.

[14] K. Yu, Q. Ai, S. Wang, J. Ni, and T. Lv, "Analysis and optimization of droop controller for microgrid system based on small-signal dynamic model," IEEE Transactions on Smart Grid, vol. 7, pp. 695-705, March 2016.

[15] X. Guo, Z. Lu, B. Wang, X. Sun, L. Wang, and J. M. Guerrero, "Dynamic phasors-based modeling and stability analysis of droopcontrolled inverters for microgrid applications," IEEE Transactions on Smart Grid, vol. 5, pp. 2980-2987, Nov 2014.

[16] B. B. Johnson, M. Sinha, N. G. Ainsworth, F. Dörfler, and S. V. Dhople, "Synthesizing virtual oscillators to control islanded inverters," IEEE Transactions on Power Electronics, vol. 31, pp. 6002-6015, Aug 2016.
[17] B. B. Johnson, S. V. Dhople, A. O. Hamadeh, and P. T. Krein, "Synchronization of nonlinear oscillators in an lti electrical power network," IEEE Transactions on Circuits and Systems I: Regular Papers, vol. 61, pp. 834-844, March 2014. 